\title{
Complicaciones obstétricas en multigestas adolescentes. Hospital Nacional Dos de Mayo 2009-20I3.
}

\author{
Jorge Renato Aparicio Ponce ${ }^{1}$, Sandra Teresa Salcedo Hermoza ${ }^{2}$
}

\section{Resumen}

Objetivo: Determinar las complicaciones obstétricas en multigestas adolescentes. Métodos: Estudio de casos y controles realizado en el Hospital Nacional Dos de Mayo del 2009 al 2013. Se realizó un análisis documental de las historias clínicas de 1,383 gestantes adolescentes. Se utilizó la prueba de chi cuadrado. Para las variables estadísticamente significativas se realizó una regresión logística multinomial. Resultados: Se admitieron 1,383 historias clínicas de gestantes adolescentes; 342 fueron multigestas adolescentes. Se presentaron 334 casos de morbilidad, siendo las más frecuentes la anemia (50,3\%), la infección vaginal $(18,7 \%)$ y la rotura prematura de membranas $(11,4 \%)$. Solo existió asociación estadísticamente significativa entre ser multigesta adolescente y la hipertensión inducida por el embarazo $(p 0,032)(\mathrm{OR}=0,54 \mathrm{IC} 95 \%[0,31-0,96])$. Conclusiones: La condición de multigesta adolescente protege en el $46 \%$ de los casos a padecer de una hipertensión inducida por el embarazo. No existe diferencia de otras morbilidades entre las primigestas y las multigestas adolescentes.

Palabras clave: Adolescente, gestación en adolescencia, complicaciones del embarazo.

\section{Abstract}

Objective: To determine the obstetric complications in multigravid adolescent. Methods: Case-control study conducted at the National Hospital Dos de Mayo from 2009 to 2013. A documentary analysis of the clinical histories of 1,383 pregnant adolescents was carried out. The chi square test was used. For the statistically significant variables, a multinomial logistic regression was performed. Results: 1,383 clinical histories of pregnant adolescents were admitted; 342 were multigravid adolescents. There were 334 cases of morbidity, the most frequent being anemia (50.3\%), vaginal infection (18.7\%) and premature rupture of membranes $(11.4 \%)$. There was only a statistically significant association between multigravida adolescent and pregnancy-induced hypertension $(p 0.032)(\mathrm{OR}=0.54$ IC 95\% [0.31-0.96]). Conclusions: The condition of multigravida adolescent protects in $46 \%$ of cases to suffer from hypertension induced by pregnancy. There is no difference from other morbidities between primigravid and multigravida adolescent.

Keywords: Adolescent, pregnancy in adolescence, complications of pregnancy.

\section{Introducción}

Las adolescentes tienen el riesgo de complicaciones durante la gestación debido a su inmadurez física y sicológica. Tienen un riesgo mayor de morir de dos a cinco veces, comparadas con gestantes en la edad ideal para concebir ${ }^{(1,2)}$. En este grupo poblacional existe mayor frecuencia de amenaza y parto pre término, hipertensión inducida por el embarazo, anemia y rotura prematura de membranas ${ }^{(3-5)}$. La frecuencia del embarazo en las adolescentes se ve que cada día aumenta; y nuestro país no escapa a este fenómeno ${ }^{(6)}$. Peor aún, es el hecho de observar el aumento de las multigestas adolescentes que son atendidas. Este problema quizá queda encubierto por la presunción a priori, que una mujer con el antecedente de haber tenido un embarazo y parto anterior representa menor riesgo en comparación con una en su primer embarazo. Las investigaciones sobre el embarazo en adolescentes, en su mayor parte son orientadas a las primigestas, tanto las complicaciones médicas y también el impacto social y sicológico. No se brinda importancia al hecho de atender una multigesta adolescente, y quizá ocurran complicaciones mayores. El objetivo del estudio fue el de determinar las complicaciones obstétricas en multigestas adolescentes.

\section{Material y métodos}

Estudio de casos y controles realizado en el Hospital Nacional Dos de Mayo, de enero de 2009 a diciembre de 2013 La población la conformaron todas las gestantes adolescentes atendidas y que culminaron la gestación en la institución. Los casos fueron las gestantes adolescentes que presentaron una o más complicaciones; y como controles las gestantes adolescentes sin complicaciones. Se seleccionaron dos con- 
troles por cada caso, creándose grupos homogéneos. Se parearon las gestantes con complicaciones con aquellas sin complicaciones de acuerdo a sí eran multigestas o primigestas, edad gestacional, nivel de instrucción, estado civil, procedencia y control prenatal. Se utilizó la técnica del Análisis Documental de las historias clínicas de las gestantes. Como Instrumento se utilizó una ficha de recolección de datos previamente elaborada. Con el programa estadístico SPSS 22.0 se creó la base de datos. Para el análisis estadístico se realizó la prueba de chi cuadrado para variables cualitativas. Las variables estadísticamente significativas fueron ingresadas en un modelo de regresión logística multinomial. Los cálculos se realizaron con un intervalo de confianza del $95 \%$.

\section{Resultados}

Del período 2009-2013 fueron admitidas al estudio 1,383 historias clínicas. De estas historias de gestantes adolescentes, 1,041 cursaban su primer embarazo y 342 ya tenían el antecedente de embarazos anteriores, considerándose multigestas adolescentes y representando el $24,7 \%$. Las características socio demográficas del estudio se aprecian en la tabla 1.

En el embarazo actual $128(37,4 \%)$ no tuvieron ningún control prenatal, $92(26,9 \%)$ tuvieron un control insuficiente, es decir menos de 6 controles, y 122 (35,7\%) tuvieron un control adecuado.

La morbilidad encontrada en el grupo de las primigestas adolescentes fue similar al grupo total de adolescentes. Este grupo presentó una morbilidad de 1,112 casos. Las tres causas más frecuentes fueron: la anemia $(50,4 \%)$, la infección vaginal $(23,3 \%)$ y la rotura prematura de membranas $(12,7 \%)$ (Tabla 2$)$. Mientras que en el grupo de las multigestas adolescentes, la morbilidad se presentó en 334 casos. Las tres primeras causas fueron: la anemia $(50,3 \%)$, la infección vaginal $(18,7 \%)$ y la rotura prematura de membranas $(11,4 \%)$. La infección urinaria $(5,3 \%)$, a diferencia del grupo de las primigestas adolescentes, fue más frecuente que la hipertensión inducida por el embarazo $(4,4 \%)$. A diferencia de las primigestas adolescentes, el desgarro perineal se presentó con más frecuencia (Tabla 3).

En cuanto a la morbilidad encontrada, el desgarro perineal fue más frecuente en las multigestas adolescentes. Se presentaron con similar frecuencia en ambos grupos la anemia $(50,3 \% / 50,4 \%)$, la infección urinaria $(5,3 \% / 5,7 \%)$, la amenaza del parto pre término $(1,5 \% / 1,7 \%)$, el parto pre término $(3,5 \% / 3,8 \%)$, y la rotura prematura de membranas fue similar $(11,4 \% / 12,7 \%)$. Fueron más frecuentes en las primigestas, la infección vaginal $(18,7 \% / 23,3 \%)$ y la hipertensión inducida por el embarazo $(4,4 \% / 7,8 \%)$ (Figura 1$)$.

\begin{tabular}{|c|c|c|}
\hline \multicolumn{3}{|c|}{ Tabla 1} \\
\hline \multicolumn{3}{|c|}{$\begin{array}{l}\text { Características socio demográficas de las multigestas adolescentes } \\
\text { en el Hospital Nacional Dos de Mayo Lima-Perú 2009-20 I3 }\end{array}$} \\
\hline CARACTERISTICA & FRECUENCIA & PORCENTAJE \\
\hline \multicolumn{3}{|l|}{ Tipo de adolescencia } \\
\hline Temprana $>10$ años pero $<$ de 15 años & 1 & 0,3 \\
\hline Tardía $>0=15$ años pero $<$ de 19 años & 341 & 99,7 \\
\hline \multicolumn{3}{|l|}{ Estado Civil } \\
\hline Soltera & 235 & 68,7 \\
\hline Casada & 2 & 0,6 \\
\hline Conviviente & 105 & 30,7 \\
\hline \multicolumn{3}{|l|}{ Grado de Instrucción } \\
\hline \multicolumn{3}{|l|}{ Ninguno } \\
\hline Primaria Incompleta & 8 & 2,3 \\
\hline Primaria Completa & 20 & 5,8 \\
\hline Secundaria Incompleta & 125 & 36,5 \\
\hline Secundaria Completa & 161 & 47,1 \\
\hline Técnica Incompeta & 3 & 0,9 \\
\hline Superior Incompleta & 1 & 0,3 \\
\hline Superior Incompleta & 1 & 0,3 \\
\hline Técnico completo & 2 & 0,6 \\
\hline TOTAL & 342 & 100,0 \\
\hline
\end{tabular}


DIAGNÓSTICO Vol. 58(I) Enero - Marzo 2019 •Complicaciones obstétricas • Aparicio Ponce JR. y col.

\begin{tabular}{|c|c|c|}
\hline \multicolumn{3}{|c|}{$\begin{array}{l}\text { Morbilidad de las primigestas adolescentes en el Hospital Nacional Dos de Mayo } \\
\text { Lima- Perú 2009-2013 }\end{array}$} \\
\hline & \multicolumn{2}{|c|}{ Primigesta (1041) } \\
\hline TIPO DE MORBILIDAD EN PRIMIGESTAS ADOLESCENTES & FRECUENCIA & PORCENTAJE \\
\hline Anemia & 525 & 50.4 \\
\hline Infección vaginal & 243 & 23.3 \\
\hline Rotura prematura de membranas & 132 & 12.7 \\
\hline Hipertensión inducida por el embarazo & 81 & 7.8 \\
\hline Infección urinaria & 59 & 5.7 \\
\hline Parto pretérmino & 40 & 3.8 \\
\hline Amenaza de parto pretérmino & 18 & 1.7 \\
\hline \multirow[t]{2}{*}{ Desgarro perineal } & 14 & 1.3 \\
\hline & 1112 & \\
\hline
\end{tabular}

\begin{tabular}{|c|c|c|}
\hline \multicolumn{3}{|c|}{$\begin{array}{l}\text { Morbilidad de las multigestas adolescentes en el Hospital Nacional Dos de Mayo } \\
\text { Lima- Perú 2009-2013 }\end{array}$} \\
\hline & \multicolumn{2}{|c|}{ Multigesta (342) } \\
\hline TIPO DE MORBILIDAD EN MULTIGESTAS ADOLESCENTES & FRECUENCIA & PORCENTAJE \\
\hline Anemia & 172 & 50.3 \\
\hline Infección vaginal & 64 & 18.7 \\
\hline Rotura prematura de membranas & 39 & 11.4 \\
\hline Infección urinaria & 18 & 5.3 \\
\hline Hipertensión inducida por el embarazo & 15 & 4.4 \\
\hline Parto pretérmino & 12 & 3.5 \\
\hline Desgarro perineal & 9 & 2.6 \\
\hline \multirow[t]{2}{*}{ Amenaza de parto pretérmino } & 5 & 1.5 \\
\hline & 334 & \\
\hline
\end{tabular}

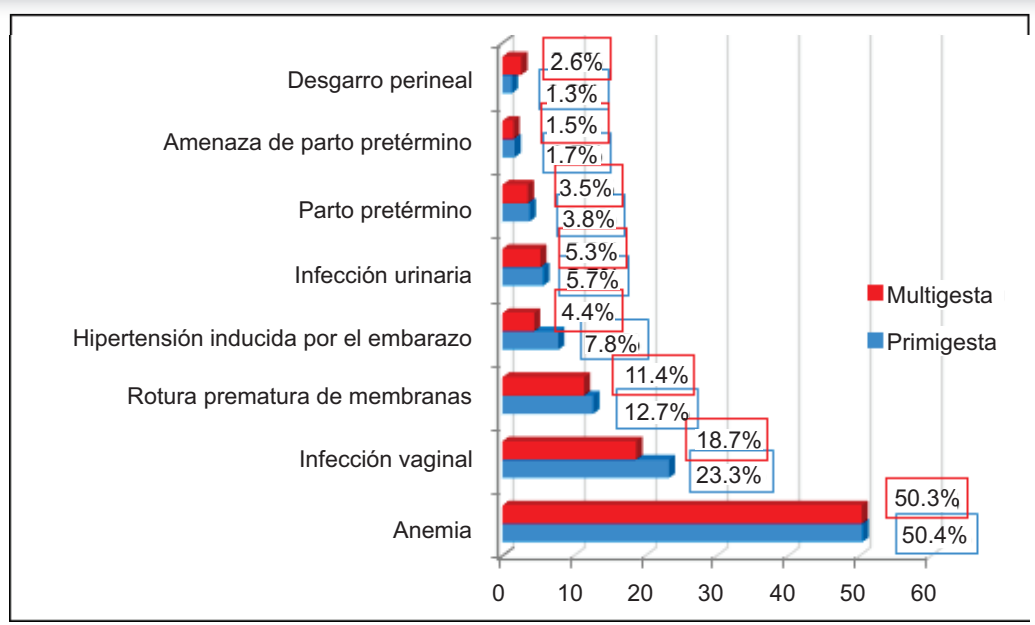

Figura 1. Comparación de la frecuencia de morbilidad entre primigestas y multigestas adolescentes en el Hospital Nacional Dos de Mayo Lima-Perú 2009-2013. 
Al buscar la asociación estadística entre gestación de las adolescentes y la presencia de complicaciones se aprecia que solo existió asociación estadísticamente significativa entre ser multigesta adolescente $\mathrm{y}$ la hipertensión inducida por el embarazo $(p 0,032)$. De esta forma, ser multigesta adolescente resultó ser un factor protector para presentar hipertensión inducida por el embarazo $(\mathrm{OR}=0,54 \mathrm{IC} 95 \%[0,31-0,96])$. Todas las demás asociaciones entre la gestación de las adolescentes y las complicaciones se pueden apreciar en la tabla 4.

En las multigestas adolescentes el embarazo culminó en aborto en el 19,0\% (65), y por parto en el 81,0\% (277) de los casos; el parto por vía abdominal fue más frecuente $(144 / 42,1 \%)$ frente a la vía vaginal $(133 / 38,9 \%)$. No se presentaron casos de embarazos ectópicos en este grupo. Hubo similitud entre las adolescentes primigestas y multigestas, referente al tipo y frecuencia de parto. Se encontró una diferencia leve para el aborto, teniendo las multigestas adolescentes una frecuencia superior, $19,0 \%$ frente a un $14,8 \%$, comparada con las primigestas. La indicación principal y más frecuente de cesárea en las primigestas adolescentes fue la estrechez pélvica (110 casos) seguida de la distocia funicular (71 casos), mientras que en las multigestas adolescentes fueron la estrechez pélvica (33 casos) seguida de la cesárea anterior (29 casos).

\section{Discusión}

En el período de 2009 a 2013 en el Hospital Nacional Dos de Mayo, del total de gestantes adolescentes admitidas, el $24,7 \%$ fueron multigestas adolescentes, que coincide con el amplio rango para este evento a nivel de Latinoamérica $(10 \%$ $45 \%$ ) reportado en los pocos estudios sobre esta población ${ }^{(7-14)}$.

El 99,7\% de las multigestas adolescentes pertenecieron al grupo de las adolescentes tardías, es decir las mayores de 15 años, hallazgo similar a lo encontrado en nuestro medio ${ }^{(7,15)}$, y en la región ${ }^{(13,14,16)}$. El adecuado control prenatal, solo fue realizado en el $35,7 \%$, coincidente con Silveira da Silva ${ }^{(16)}$, y muy diferente a lo reportado por Claros $^{(7)}$, en la que se menciona que el $63,0 \%$ fueron gestantes controladas.

En la población de gestantes adolescentes en el período de estudio no se registraron casos de muerte materna. Esto en relación con el nivel resolutivo de la institución y también, a quizá la referencia oportuna de una gestante adolescente considerada de alto riesgo; la cual debe ser controlada en un servicio de esa envergadura, para evitar eventos adversos por una mala accesibilidad de atención de salud ${ }^{(3,9)}$.

\begin{tabular}{|c|c|c|c|c|c|c|}
\hline \multicolumn{7}{|l|}{ Morbilidad de las } \\
\hline \multirow{2}{*}{ MORBILIDAD } & \multicolumn{6}{|c|}{ Multigesta adolescente } \\
\hline & & Si & No & & valor $p$ & OR (IC 95\%) \\
\hline \multirow[t]{2}{*}{ Anemia } & $\mathrm{Si}$ & 172 & 525 & 697 & 0.694 & $0,99(0,78-1,28)$ \\
\hline & No & 170 & 516 & 686 & & \\
\hline \multirow[t]{2}{*}{ Infección vaginal } & $\mathrm{Si}$ & 64 & 243 & 307 & 0.074 & $0,76(0,56-1,03)$ \\
\hline & No & 278 & 798 & 1076 & & \\
\hline \multirow{2}{*}{$\begin{array}{l}\text { Rotura prematura de } \\
\text { membranas }\end{array}$} & $\mathrm{Si}$ & 39 & 132 & 171 & 0.534 & $0,89(0,61-1,30)$ \\
\hline & No & 303 & 909 & 1212 & & \\
\hline \multirow{2}{*}{$\begin{array}{l}\text { Hipertensión inducida por } \\
\text { el embarazo }\end{array}$} & $\mathrm{Si}$ & 15 & 81 & 96 & 0.032 & $0,54(0,31-0,96)$ \\
\hline & No & 327 & 960 & 1287 & & \\
\hline \multirow[t]{2}{*}{ Infección urinaria } & $\mathrm{Si}$ & 18 & 59 & 77 & 0.777 & $0,92(0,54-1,59)$ \\
\hline & No & 324 & 982 & 1306 & & \\
\hline \multirow[t]{2}{*}{ Parto pretérmino } & $\mathrm{Si}$ & 12 & 40 & 52 & 0.778 & $0,91(0,47-1,76)$ \\
\hline & No & 330 & 1001 & 1331 & & \\
\hline \multirow[t]{2}{*}{ Amenaza de parto pretérmino } & $\mathrm{Si}$ & 5 & 18 & 23 & 0.738 & $0,84(0,31-2,29)$ \\
\hline & No & 337 & 1023 & 1360 & & \\
\hline \multirow[t]{2}{*}{ Desgarro perineal } & $\mathrm{Si}$ & 9 & 14 & 23 & 0.106 & $1,98(0,85-4,62)$ \\
\hline & No & 333 & 1027 & 1360 & & \\
\hline
\end{tabular}


La morbilidad encontrada en el estudio en toda la población gestante adolescente, evidenció que la anemia fue la complicación más frecuente $(50,4 \%)$; hallazgo similar a los estudios realizados en la región ${ }^{(4-5,9,17)}$, donde además de la anemia también es frecuente la amenaza y parto pre término, la hipertensión inducida por el embarazo y la rotura prematura de membranas. Sin embargo, si bien coinciden en que la rotura prematura de membranas $(12,4 \%)$ y quizá la hipertensión inducida por el embarazo $(6,9 \%)$, son frecuentes, difieren en cuanto a la amenaza de parto pre término $(1,7 \%)$ y el parto prematuro $(3,8 \%)$, ya que su frecuencia fue baja en el estudio realizado. También la frecuencia de presentación tanto de infección urinaria ${ }^{(18)}$, como del desgarro perineal ${ }^{(19)}$, coinciden con los hallazgos de la región en su baja frecuencia. La infección vaginal fue la segunda complicación en frecuencia en el estudio $(23,3 \%)$, sin embargo esto difiere de lo reportado en la región ${ }^{(3,18-}$

A nivel nacional, las complicaciones maternas que se presentan con mayor frecuencia en las adolescentes son la anemia, la infección urinaria y la hipertensión inducida por el embarazo $^{(8,21)}$. Solo la anemia tiene una similitud con dichos hallazgos reportados, ya que la hipertensión inducida por el embarazo, y la infección urinaria, presentaron baja frecuencia. Con menor frecuencia se reportan el parto prematuro, la infección vaginal, la rotura prematura de membranas y los desgarros perineales ${ }^{(8)}$. En relación a este último grupo de complicaciones, los resultados difieren en cuanto a la infección vaginal y la rotura prematura de membranas, encontrándose estos eventos con frecuencia. Ambas patologías se reconocen que tienen relación entre ellas, sobretodo la infección vaginal como un factor de riesgo asociado, pero no imprescindible, para que ocurra la rotura prematura de membranas; y tanto en el presente estudio como en los mencionados a nivel nacional guardan relación en cuanto a sus frecuencias halladas. La disyuntiva surge en explicar por qué en el estudio las frecuencias son tan altas. Pero la asociación es demostrada por los hallazgos de ambas de manera similar en frecuencia de presentación.

Prácticamente no existen estudios de la morbilidad que presentan las multigestas adolescentes, tanto a nivel nacional como en la región, a excepción de lo reportado por Klerman ${ }^{(22)}$ mencionando para este grupo una mayor frecuencia del parto pre término. Así mismo, Martínez ${ }^{(13)}$, reporta como las complicaciones más frecuentes a la anemia $(36,0 \%)$ y la infección urinaria $(29,0 \%)$. Ambos resultados difieren a los hallados en el estudio, si bien la anemia fue la complicación más frecuente en el presente estudio, su frecuencia es mayor, encontrándose un 50,3\% de multigestas adolescentes afectadas. En cuanto a la infección urinaria, la frecuencia encontrada fue mucho menor, con solo un 5,3\%. Los resultados obtenidos demuestran una similitud en la frecuencia de la morbilidad, exceptuando el desgarro perineal y la hipertensión inducida por el embarazo al comparar el grupo de las primigestas con las multigestas adolescentes.

En el caso del desgarro perineal, se presentó con el doble de frecuencia en las multigestas adolescentes que en las primigestas $(2,6 \%$ vs $1,3 \%)$. Esto en relación a que se supone que un canal dilatado por una o más expulsiones vaginales, tiene menor posibilidad de desgarrarse en un siguiente parto. Pero esto, asumiendo que en las multigestas haya el antecedente de uno o más partos vaginales, dato no establecido en el estudio. Puede haber ocurrido que en las multigestas adolescentes los embarazos anteriores no hayan culminado en parto, comportándose como primíparas propensas a desgarros. Esta diferencia de la presentación del desgarro no fue estadísticamente significativa $(p 0,106)$.

En lo referente a la hipertensión inducida por el embarazo, está se presentó en las multigestas adolescentes, con una frecuencia de casi la mitad de los casos presentados en las primigestas adolescentes $(4,4 \%$ vs $7,8 \%)$. Esta diferencia si fue estadísticamente significativa $(p 0,032)$, y fue la única complicación que presentó este resultado estadístico a diferencia de todas las demás. Además, el análisis estadístico, OR (IC 95\%) 0,54 (0,31-0,96), demuestra que el hecho de ser una multigesta adolescente, protege en el $46 \%$ de los casos para presentar la complicación de hipertensión inducida por el embarazo. Está condición se asocia a que los extremos de la vida reproductiva, representan un factor de riesgo. Sin embargo, también se menciona la nuliparidad como un factor de riesgo para desarrollar la complicación. Por lo tanto, a pesar de que las multigestas adolescentes por la edad tienen riesgo, el hecho de ser multigestas las protegería para que no se presente ésta complicación.

Finalmente, la cesárea se presentó en el $44,4 \%$, lo que difiere de la frecuencia reportada entre $25 \%$ a $38 \%(3,9,10)$. En el grupo de las multigestas adolescentes la vía abdominal fue la más frecuente $(144 / 42,1 \%)$ frente a la vaginal $(133 / 38,9 \%)$, lo que difiere de los estudios en los que el parto vaginal se presenta con mayor frecuencia en las multigestas adolescentes ${ }^{(11,16)}$, pero coinciden con lo reportado por Oliveira de Azevedo ${ }^{(10)}$. Esto puede tener relación a que el principal diagnóstico para la cesárea fue el de cesareada anterior, lo que explica en cierta forma la elevada frecuencia de este procedimiento en este grupo.

Con el estudio se concluye que existe diferencia estadísticamente significativa en la presentación de la hipertensión inducida por el embarazo $(p=0,032)$ entre las multigestas adolescentes y las primigestas adolescentes. La multigesta adolescente, es una condición que protege en el $46 \%$ de los casos a padecer de una hipertensión inducida por el embarazo.

\section{Referencias bibliográficas}

1. Cabezas E. Mortalidad materna y perinatal en adolescentes. Rev Cubana Obstet Ginecol. 2002;28(1):5-10.

2. Olukoya A, Kaya A, Ferguson B, AbouZahr C. Unsafe abortion in adolescents. Int J Gynaecol Obstet. 2001;(75):137147.

3. Acevedo M, Gómez D, Arbelo D, Rodríguez I. Morbilidad por

embarazo en la adolescencia en el hogar materno municipal Tamara Burke. MEDISAN. 2010;14(7):976-981.

4. Murillo O, Zea M, Pradilla A. Situación nutricional de la gestante y su recién nacido en Cali, 2008. Rev salud pública. 2011;13(4):585-596.

5. Ortega, P, Leal, J, Chávez C, Mejía L, Chirinos N. Transición 
alimentaria y anemias nutricionales en adolescentes femeninas no gestantes y gestantes. Revista de la Universidad del Zulia $3^{\circ}$ época. Ciencias exactas, Naturales y de la Salud. 2011;2(3):99117.

6. Instituto Nacional de Estadística e Informática. Perú. Encuesta Demográfica y de Salud Familiar-ENDES continua, 2009. Visión Nacional y Departamental. Lima. 2010.

7. Claros A. Adolescentes multigestas: aspectos socioculturales. Paedriática. 2006;8(1):15-19.

8. Castillo O, Mariños E. Morbilidad obstétrica de la gestante adolescente en el Hospital Docente Madre Niño San Bartolomé, Enero a Diciembre, 2004. An Fac Med Lima. 2006;(67) Suppl 1 S86.

9. Bojanini J, Gómez J. Resultados obstétricos y perinatales en adolescentes. Revista Colombiana de Obstetricia y Ginecología. 2004;55(2):114-121.

10. Oliveira de Azevedo V, Costa M, Queiroga F, Brandão A, Marinho, Mendes R. Via de parto em gestacoes sucessiva sem adolescentes: estudo de 714 casos. RBGO. 2004;26(9):703707.

11. Persona L, Kaku, y Tarallo M. Perfil de adolescentes com repeticao da gravidez atendidas num ambulatório de pré - natal. Latinoam Enfermagem. 2004;12(5):745-750.

12.Ximenes F, Marques, Rocha J. Problemas vivídos por las adolescentes durante la gestación. Enfermería Global Revista Electrónica. 2008;7(1):1-12. Recuperado el 01 de Marzo de 2013, de http://revistas.um.es/eglobal/article/view/832/2881

13. Martinez S. Factores que influyen en la multiparidad en adolescentes de 14 a 19 años, atendidas en la consulta externa del Hospital Materno Infantil Dra. Matilde Hidalgo de Procel, en el período del 06 de setiembre del 2012 a febrero 2013. Guayaquil.

14.Idrobo I. (2010). Prevención del embarazo subsecuente en adolescentes. Centro de Salud de Catamayo. Guayaquil.

15. Diego P, Huarcaya G. (2008). Factores familiares y reproductivos asociados al embarazo reincidente en adolescentes-Instituto Nacional Materno Perinatal, 2008. (Tesis para obtener el Título de Licenciada en Obstetricia). Recuperado el 11 de Marzo de 2013, de http://www. cybertesis.edu.pe/sisbib/2008/diego_dp/pdf/diego_dp.pdf

16. Silveira da Silva K, Rozenberg R, Bonan C, Costa Chuva V, Furtadoo da Costa S, Souza M. Gravidez recorrente na adolescencia e vulnerabilidade social no Rio de Janeiro (rj, brasil): uma análise de dados do sistema de nascidos vivos. Ciencia \& Saúde Coletiva. 2011;16(5):2485-2493.

17. Conde A, Belizan L. Maternal perinatal morbility and mortaliy associated with adolescent pregnancy in Latinamerica: cross sectional study. Am J Obstet Gynecology. 2005;(192):342-349.

18. Manrique R, Rivero A, Ortunio M, Rivas M, Cardozo R, Guevara H. Parto pre término en adolescentes. Rev Obstet Ginecol Venez. 2008;68(3):144-149.

19.Sáez Cantero V. Embarazo y adolescencia. Resultados perinatales. Rev Cubana ObstetGinecol. 2005;31(2). Recuperado el 01 de Marzo de 2013, de http://scielo.sld. $\mathrm{cu} /$ scielo.php? $\mathrm{pid}=\mathrm{S} 0138-600 \mathrm{X} 2005000200001$ \& script $=$ sci abstract

20. Sáez Cantero V. (2005). Embarazo y adolescencia. Resultados perinatales. Rev Cubana ObstetGinecol, 31(2). Recuperado el 01 de Marzo de 2013, de http://scielo.sld.cu/scielo.php?pid=S0 138-600X2005000200001\&script $=$ sci abstract

21. Choque F. (2003). Factores de riesgo obstétrico en el embarazo de adolescentes. (Tesis para optar al Título de especialista en Ginecología y Obstetricia), Universidad Nacional Mayor de San Marcos, Lima, Perú. Recuperado el 01 de Marzo de 2013, de http://www.cybertesis.edu.pe/sisbib/2004/choque_mf/ $\mathrm{html} /$ index-frames.html

22. Klerman L. (2004). Another Chance: Preventing Additional Births to Teen Mothers. Wasington DC: TheNational CampaigntoPreventTeenPregnancy. 